\title{
Application of Recycle Composites on Cold-rolled Ribbed Roller
}

\author{
Y. P. SONG ${ }^{1, *}$, a , W. Q. LI ${ }^{2}$, L. YOU ${ }^{1}$ and X. D. HUANG \\ ${ }^{1}$ Mat. Sci. \& Eng. College, Henan University of Science and Technology, No 263 Kaiyuan Road, \\ Luoyang 471023, China \\ ${ }^{2}$ Luoyang Jinfeng Casting Co. Ltd, No. 258 Heluo Road, Luoyang 471000, China \\ asypei@haust.edu.cn \\ *Corresponding author
}

Keywords: Reject Metal Matrix Composites, Recycled Roller, Microstructure and Properties, Application

\begin{abstract}
Reject metal matrix composites parts reclaimed from industrial market were re-melted in a medium frequency induction furnace. A composites recycled roller used for cold-rolled wire mill was fabricated by centrifugal casting. The properties, microstructures and application of the recycled roller were investigated. The results show that the recycled roller is a composite structure consisting of outer recycled composite layer reinforced with remaining carbide particle containing $\mathrm{W}$ in re-melting and inner matrix. The carbide particle was evenly distributed in the composite layer of $15-18 \mathrm{~mm}$ in thickness and volume fraction is approximately 65-70 vol. \%; hardness attained to HRA81-84 in the composite layer, and HRA74-76 in inner matrix where the toughness was over 6.0 $\mathrm{J} / \mathrm{cm}^{2}$; the wear resistance and service life of the recycled roller and the original composites roller are almost same when same carbide volume fraction were obtained, but the production cost of the recycled roller decreased by $25 \%$.
\end{abstract}

\section{Introduction}

With the development of high-speed rolling technology, work rolls used for cold-rolling mill are demanded to meet requirements of a long service life, low costs and stable operation ${ }^{11]}$. While cemented carbide roller produced through powder metallurgy possess excellent wear resistance and hardness ${ }^{[2]}$. However, besides high costs, the such rollers are prone to cracking in service due to low toughness, which causes massive waste of raw materials and energy ${ }^{[3]}$. Consequently, the mechanical properties and technology costs of the work roller for rolling mills are of great concern to the roller designers and operators.

Particle-reinforced metallic matrix composites (PRMMC) have been paid much attention due to its excellent mechanical and thermal properties ${ }^{[4]}$. Recently, our research team has developed a new type of roller rings made from a PRMMC by means of centrifugal casting technique ${ }^{[5,6]}$. The mechanical properties of the PRMMC roller rings exceed that of the cemented carbide roller, and the production cost is $50 \%$ of the cemented carbide roller rings. However, the accumulated reject roller rings in the industrial dump sites can not be reused, which will cause a lot of waste of energy sources and roller materials. Therefore, it is necessary to evaluate the technological feasibility of reused the reject roller rings.

In this study, a recycled cold-rolled ribbed roller was manufactured by remelting of the failure metal matrix composites parts and centrifugal casting technique. The aim of the work is to investigate the effect of re-melting technology on microstructures and properties of the recycled roller and its application in metallurgical industry.

\section{Experimental Procedures}

The failure roller rings made by metal matrix composites (original composites) were attained from industrial waste market. The chemical composition (expressed in weight) of the matrix alloy for the failure roller rings is as follows:3.2- 3.4\% C, 2.3-2.5\% $\mathrm{Si}, 3.5-4.0 \% \mathrm{Ni}, 0.25-0.5 \% \mathrm{Mo}, \mathrm{S}$ and $\mathrm{P}<$ 
$0.03 \%$ and others Fe, and the volume fraction of the tungsten carbide for reinforcing is about $75 \%$. The re-melting of the reject metal matrix composites parts which were cut into pieces was carried out in a medium frequency induction furnace. When the melts temperature reached about $1650{ }^{\circ} \mathrm{C}$, it was poured into a ladle with Re-Mg nodularizer, and then into the mold rotating at $850 \mathrm{rpm}$ (centrifugal casting). A composite structure recycled roller ring, consists of outer recycled composites layer reinforced with remaining carbide particle containing $\mathrm{W}$ in re-melting and inner Fe-C alloy layer, was obtained in this process. The dimensions of the recycled rollers are as follows: $234 \mathrm{~mm}$ in outer diameter, $124 \mathrm{~mm}$ in inner diameter, and $90 \mathrm{~mm}$ in length.

The specimens of the recycled roller ring for metallographic, mechanical properties and wear tests were cut off by line cutting method. The impact and hardness tests are carried out on a JB147/294A type impact tester and HR150D hardness tester, respectively. The size of the un-notched impact specimens is $10 \mathrm{~mm} \times 10 \mathrm{~mm} \times 55 \mathrm{~mm}$. Dry sliding wear tests at room temperature are carried out using a pin-on-disc wear tester which employed a steel disc rotating against the test pin on which a load applied by a lever mechanism. The sliding wear speed and load applied were 40 $\mathrm{m} / \mathrm{s}, 60 \mathrm{~m} / \mathrm{s}$ and $50 \mathrm{~N}, 100 \mathrm{~N}, 150 \mathrm{~N}$, respectively. The weight loss of the test pin is determined by a scale with an accuracy of $10-4 \mathrm{~g}$ at definite time intervals.

All specimens for microstructure observation were prepared by standard metallographic polishing techniques. The microstructures and micro-composition of the recycled ring were analyzed by scanning electron microscopy (SEM) with energy dispersive spectroscopy (EDS) .

\section{Results and Discussion}

\section{Mechanical Properties}

Table 1 presents the mechanical properties of the composites recycled roller and original composites roller. As shown in Table 1, the impact toughness both in outer recycled composites-layer and inner Fe-C alloy matrix of the composites recycled roller is less than those of the original composites roller, but it exceeds those of the hard alloy rolls $\left(2.5-3.5 \mathrm{~J} / \mathrm{cm}^{2}\right)$. The hardness of composites working-layer in the recycled roller ring is almost the same as those of the original composites roller ring. Therefore, the composites recycled roller rings can be expected to meet the requirements of rollers used in cold-rolled ribbed rolling mill due to their high hardness in working layer and good impact toughness in inner matrix.

Table 1 Mechanical property of the recycled composites and original composites roller

\begin{tabular}{|c|c|c|c|}
\hline Material of roller ring & Impact toughness $\left(\mathrm{J} / \mathrm{cm}^{2}\right)$ & Hardness & (HRA) \\
\hline Recycled composites work-layer & $3.5-4.6$ & & $81-84$ \\
\hline Inner alloy matrix for the recycled roller & $6.0-6.9$ & & $74-76$ \\
\hline Original composites work layer & $4.3-5.2$ & & $80-85$ \\
\hline Inner alloy matrix for the original composites roller & $8.2-11.5$ & & $72-74$ \\
\hline
\end{tabular}

\section{Microstructures}

Figure 1 shows the distribution of remaining tungsten carbide particle in working-layer and the inner matrix microstructure of the composites recycled roller ring. It can be seen from Fig. 1 that the remaining tungsten carbide particle in re-melting are evenly distributed in the recycled composites working layer, and their volume fraction reaches up to 65-70\% (Fig.1a). The joint interface between the inner Fe-C alloy matrix and recycled composites working layer is clear and displays linear shape (Fig.1b). The microstructure of inner Fe-C alloy matrix consists of bainite, graphite and rod-like or block-like crystallites (Fig.1c). The EDS analyses results show that these crystallites are complex carbides containing W, C and Fe (Fig.1d). Tungsten (W) is found in the inner $\mathrm{Fe}-\mathrm{C}$ alloy matrix for the recycled roller ring because the carbides containing $\mathrm{W}$ in the 
original composites partially dissolved into the inner $\mathrm{Fe}-\mathrm{C}$ alloy matrix during re-melting process (Fig.1e).

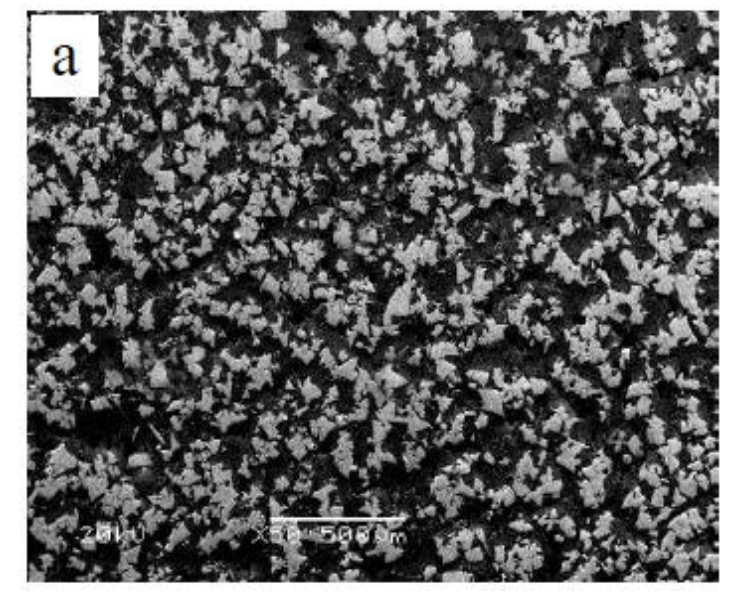

a. Distribution of remaining carbide particle in the composites layer

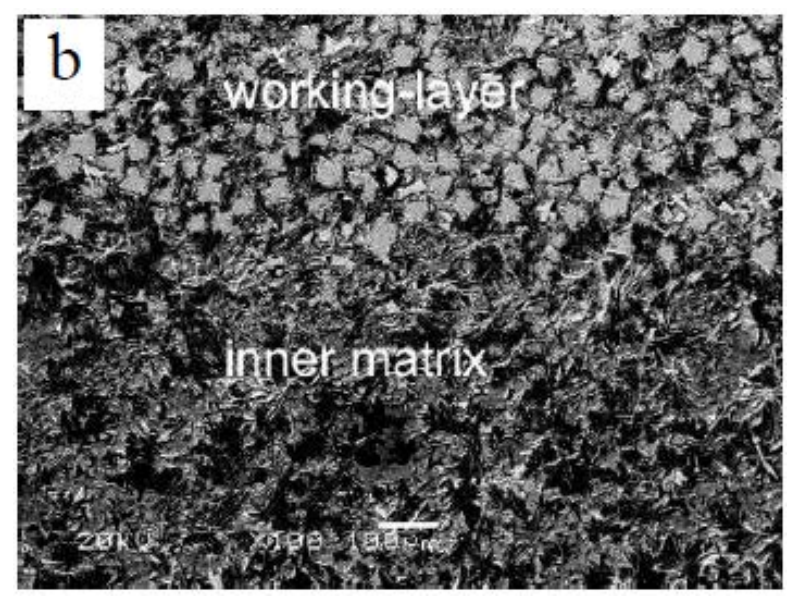

b. Interface bonding between core alloy matrix and composites working-layer

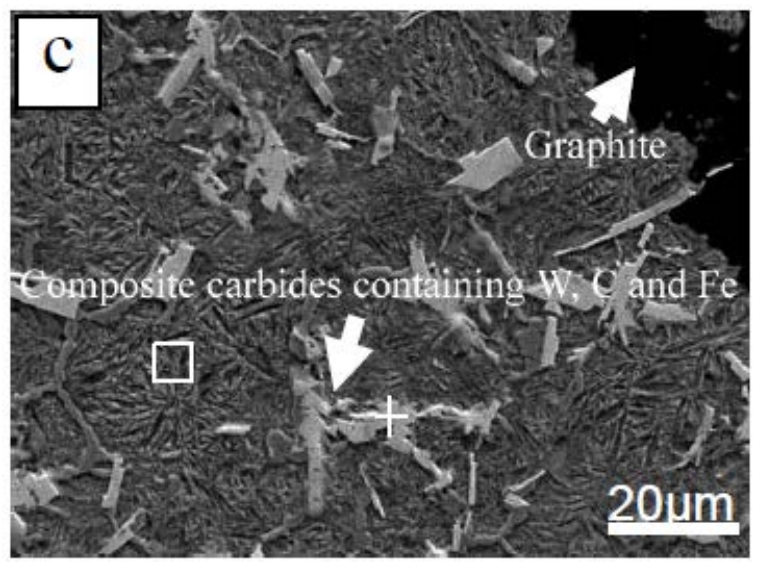

c. Microstructure of core Fe-C alloy matrix 


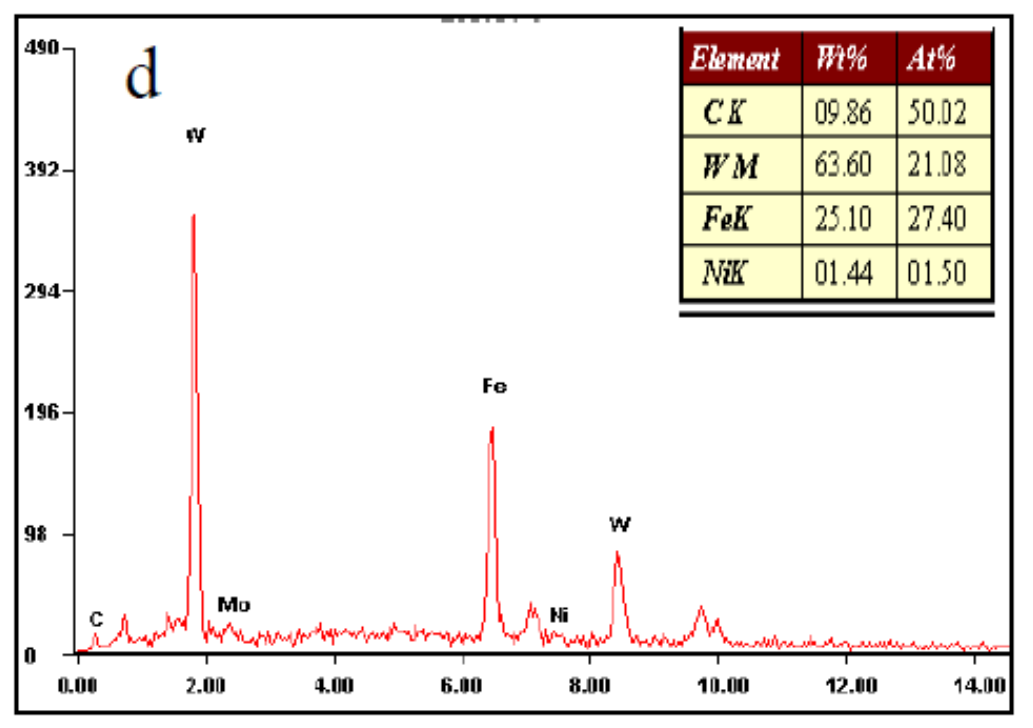

d. EDS analyses of the complex carbide

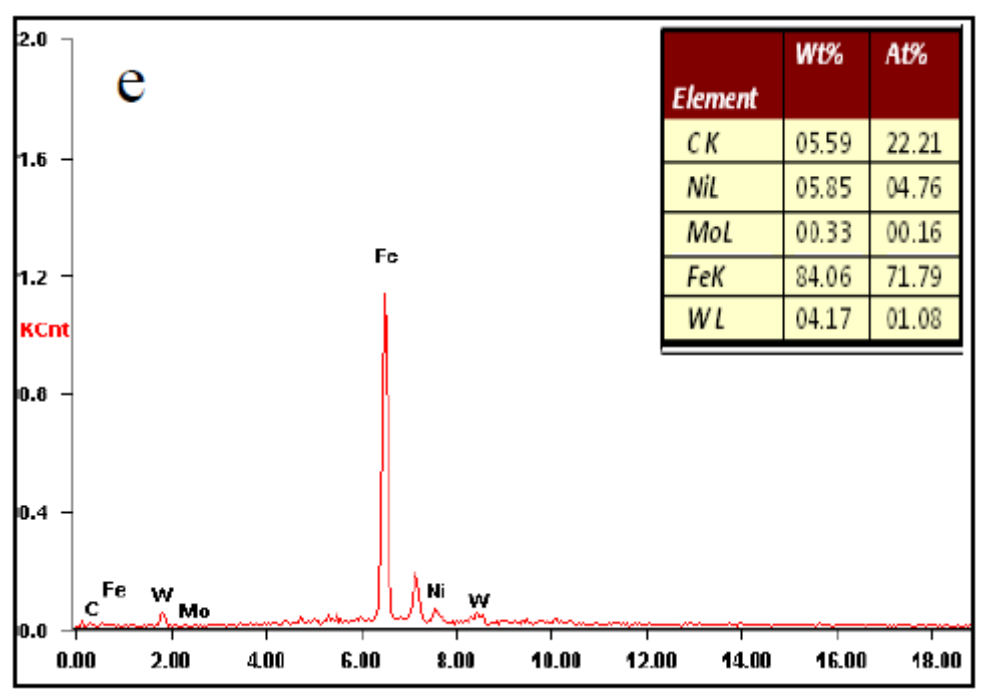

e. EDS analyses of the inner Fe-C alloy matrix

Figure1. Microstructure and EDS analyses of the recycled composites rollers:

\section{Wear Property}

Figure 2 shows the dry sliding wear rate at room temperature for the recycled composites and the original composites under various loads and sliding speeds. It can be observed from Fig. 2 that under the sliding speeds of $40 \mathrm{~m} / \mathrm{s}$ and $60 \mathrm{~m} / \mathrm{s}$, the wear rates of the recycled composites and original composites reaches $3.51 \times 10^{-6} \mathrm{~g} / \mathrm{m}, 3.5 \times 10^{-6} \mathrm{~g} / \mathrm{m}$ and $4.34 \times 10^{-6} \mathrm{~g} / \mathrm{m}, 4.22 \times 10^{-6} \mathrm{~g} / \mathrm{m}$ at the load of $50 \mathrm{~N}$, and the wear rates exhibit a nearly linear increase with the loads. When the given load increases up to $150 \mathrm{~N}$ from $50 \mathrm{~N}$, the wear rates of the two composites at the sliding speeds of $40 \mathrm{~m} / \mathrm{s}$ and $60 \mathrm{~m} / \mathrm{s}$ increase to $12.38 \times 10^{-6} \mathrm{~g} / \mathrm{m}, 12.27 \times 10^{-6} \mathrm{~g} / \mathrm{m}$ and $21.23 \times 10^{-6} \mathrm{~g} / \mathrm{m}, 20.11 \times 10^{-6} \mathrm{~g} / \mathrm{m}$, respectively. Comparatively, the wear rate of the recycled composites seems a little higher than that of the original composites under the load of $150 \mathrm{~N}$ and sliding velocity of $60 \mathrm{~m} / \mathrm{s}$. The reason for this is the fact that under smaller given loads and sliding speed, the temperature of wear surface for the recycled composites testing pin is lower, the lower temperature of the wear surface under the low wear speed and loads slows down the rates of oxide formation and removal, and the remaining tungsten carbides in the recycled composites by preventing metal-to-metal contact at the wear surface decreases the wear rate of the testing pin. When the wear speed and load increases up to 60 $\mathrm{m} / \mathrm{s}$ and $150 \mathrm{~N}$, the wear surface temperature of the recycled composites testing pin rises in wear 
process, and it will lead to generation of oxide films which are formed by the oxidation of the iron substrate itself on the wear surface of the pins. And this pressure of high load would cause matrix softening of the recycled composites, which would result in the carbide particle being pushed below the wearing surface. Metal-to-metal contact makes the oxide film grow rapidly. The oxide film is fractured under the action of friction force to occur the peeling of oxide film. Rapid wear is occurred between the pin sample and the counter face. So the wear rate is increased. Increasing the volume fraction of carbide particle in the composites can reduce Fe-C alloy matrix on wear surface of the pin contact with the disc, slow down the rate of oxide formation and removal. Accordingly, the wear rate of the recycled composites seems a little higher than that of the original composites under same testing condition due to volume fraction of the remaining tungsten carbide in the recycled composites (65-70 vol.\%) below that of the original composites (75 vol.\%).
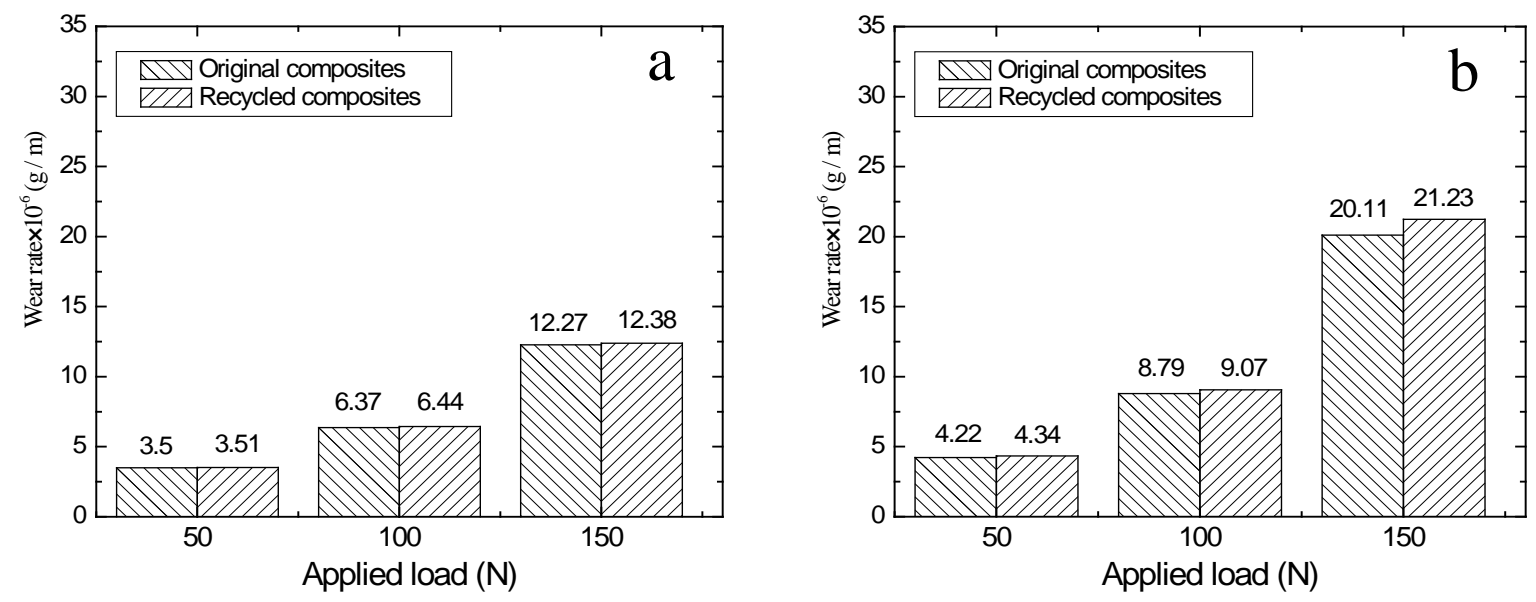

Figure 2 Wear rate comparison of the recycled composites and original composites under the same applied load: a. Wear speed of $40 \mathrm{~m} / \mathrm{s}$; b. Wear speed of $60 \mathrm{~m} / \mathrm{s}$

\section{Application}

Taking cold-rolled ribbed roller as application objects, a composites recycled roller ring (outer diameter $232 \mathrm{~mm}$, inner diameter $110 \mathrm{~mm}$, highness $78 \mathrm{~mm}$ ) are fabricated by centrifugal casting method at $850 \mathrm{rpm}$. After tempering of $310^{\circ} \mathrm{C}$, a cubic boron nitride cutting tools is used for rough machining of the composites recycled roller roughs, and then diamond grinding wheel is used for finish machining (see Fig. 3). The working-layer thickness of $18 \mathrm{~mm}$ recycled composites can satisfy the required radial usage requirements of the cold-rolled wire mill roller rings. The composites recycled roller rings are installed in the cold-rolled ribbed rolling mill to roll $\varphi 10$ ribbed wires, after three days' continuous use, the rolling quantity for the ribbed wires on one groove pass reach $3210 t$, and reach or exceed the rolling quantity of the original composites rollers which are about 3180-3240t in same service condition, approached to that of the hard alloy roller (328-3315t) when they are both of the same carbide volume fractions in working-layer. Compared with the original composites roller, the production cost of the composites recycled roller decreases by $25 \%$. 

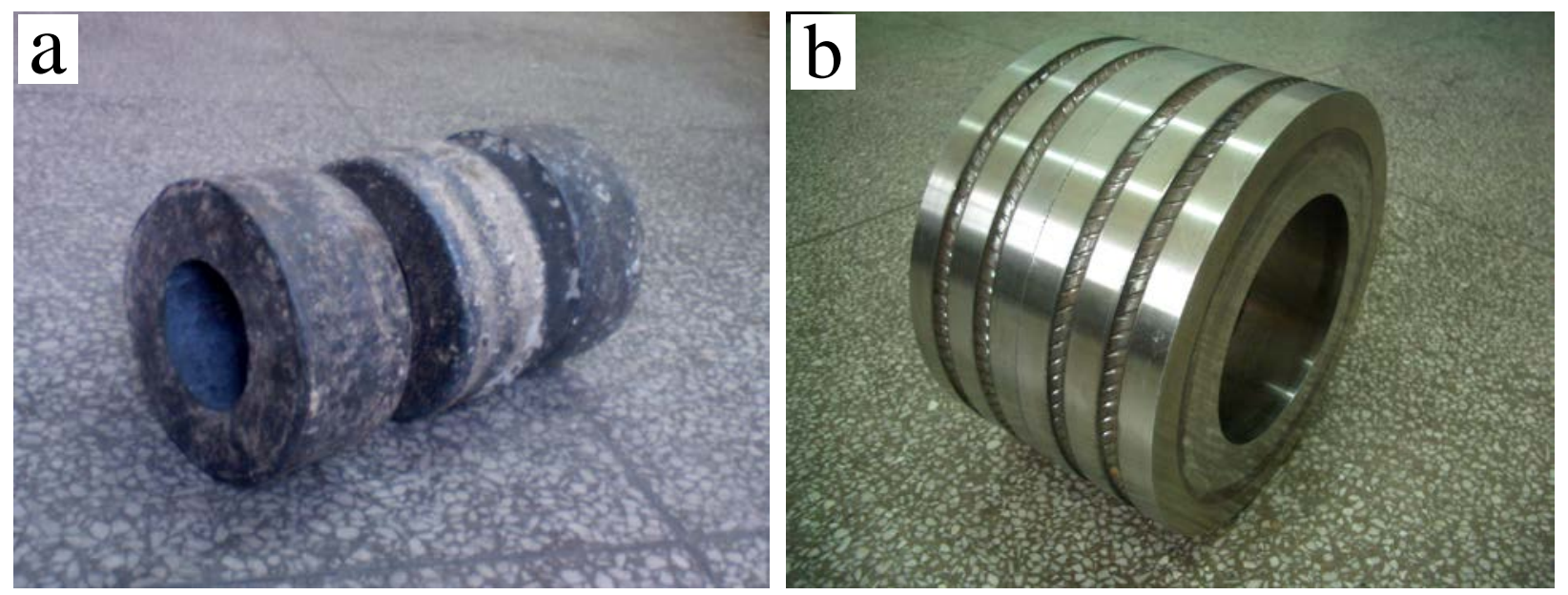

Figure3 Composites recycled rollers fabricated by centrifugal casting. (a) Rough casting and (b)

Roller after installing use

\section{Research Highlights}

$>$ Reject metal matrix composites (origin composites) parts from industrial waste market were re-melted in a medium frequency induction furnace, a composite recycled roller was fabricated with the re-melts for the first time.

$>$ Investigation of Microstructures, mechanical properties and wear characteristics for the composites recycled roller was carried out by SEM, EDS and mechanical property tester.

$>$ Usability of the composites recycled roller ring in cold-rolled wire mill was evaluated by practical application.

\section{Conclusion}

(1) A composites recycled roller, consist of outer recycled composites layer and inner alloy layer, is manufactured by centrifugal casting technology. The remaining tungsten carbide particle in re-melting were uniformly distributed in outer recycled composites layer of $15-18 \mathrm{~mm}$ in thickness and their volume fraction reached $65-70$ vol.\%.

(2) The hardness of composites recycled roller surface working-layer and core matrix were kept at HRA81-84 and HRA74-76, respectively. The impact toughness of core matrix exceeded 6J/cm2, and less than those of the original composites rollers.

(3) The wear rates of the recycled composites increase with the load at the two sliding speed. Under same load, the wear rates of the recycled composites are little more than that of the original composites.

(4) The service life of the composites recycled roller using for cold-rolled ribbed wire rolling mill was almost the same as that of original composites roller under same carbide volume fraction, but the production cost of the composites recycled roller decreased by $25 \%$.

\section{References}

[1] M. Pellizzari, D. Cescato, M. G. De Flora, 'Hot friction and wear behaviour of high speed steel and high chromium iron for rolls’: Wear, 2009, 267, 467-475.

[2] R. González, J. Echeberría, J. M. Sánchez, F. Castro: 'WC-(Fe,Ni,C) hardmetals with improved toughness through isothermal heat treatments', J. Mater. Sci., 1995, 30, 3435-3439.

[3] B. Qu, S. G. Zhu, W. S. Gu: 'Roll Failure Fashion and It's Causes Analysis', Shandong Metallurgy, 2005, 27(1), 31-33. 
[4] J. Wang, Y.S. Wong: 'In-situ production of Fe-TiC composite', Mater. Lett., 2007, 61, 4393-4395.

[5] Y. P. Song, H. Yu, X. M. Mao: 'Wear Behavior of WC $\mathrm{C}_{\mathrm{P}} / \mathrm{Fe}-\mathrm{C}$ Composites Under High Speed Dry Sliding', J. Mater. Sci., 2008, 43, 2686-2692.

[6] Y. P. Song, H. Yu, J. G. He: 'Elevated temperature sliding wear behaviour of WC-reinforced ferrous matrix composite’, J. Mater. Sci., 2008, 43, 7115-7120. 